\title{
Diet, Microbiome and Multiple Sclerosis
}

\section{(ㄷ) (i) (오 $\ominus$}

Authors

Aiden Haghikia ${ }^{1}$, Ralf Linker ${ }^{2}$

Affiliations
1 Neurologische Klinik der Ruhr-Universität Bochum,
St. Josef-Hospital
2 Neurologische Klinik der Friedrich-Alexander-Universität
Erlangen

Key words

microbiome, diet, MS, gut

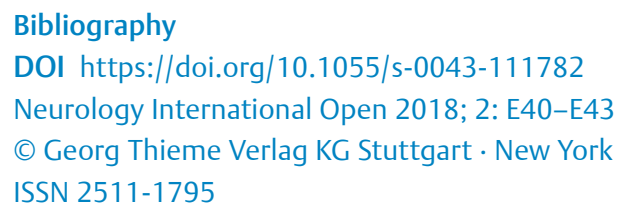

\begin{abstract}
During recent years, research into the causes of multiple sclerosis (MS), especially in the field of genetic risks of this disease, has made immense advances, thanks to new molecular biology techniques and large consortia. However, the functional relevance of disease-associated risk gene variants remains unclear, and representing about one-third of the overall risk compared to environmental risk factors, plays a smaller role. Thus, the further identification and, where appropriate, prevention of environmental risks is currently the focus of research. Here recently, risk factors for MS have been shown which are closely related to Western diet and lifestyle, such as high consumption of salt and cigarette smoking. In addition, the human gut continues to be the focus of research: with its large surface, the gut offers immense opportunities for interaction between intestinal bacteria, food and their metabolic products, as well as the immune system associated with the gut. In the present paper, we summarize the findings of the constantly growing research field dealing with the gut, nutrition and MS, and discuss how these findings, as shown recently for fatty acids, could have translational-therapeutic potential.
\end{abstract}

\section{Introduction}

The British natural scientist Sir Francis Galton (1822-1911) coined the phrase "nature and nurture", that is, inherent (nature) as well as acquired (nurture) factors affect a person's destiny [1]. What was originally conceived as a concept to explain personality and character traits of people can also be applied to various diseases in neurology. A good example of this is multiple sclerosis (MS).

According to current hypotheses, MS is considered an autoimmune disease of the central nervous system transmitted by T cells. Despite extensive research in recent decades, the exact pathogenesis and etiology of the disease is still not fully understood [2]. Since MS is an autoimmune disease with many causative factors, genetics is a constituent in the origin of the disease, but environmental influences likewise play a role. It is known from twin studies that genes represent about $1 / 3$ of the causative factors, but environmental factors pose $2 / 3$ of the predilective components and thus form the greater part of the risk for the development of MS. A distinction can be made between modifiable and non-modifiable risk factors for MS. For many years focus was largely on non-modifiable factors, such as a family history of MS, fe- male gender, age ( $80 \%$ have an onset between the 20 th and 40 th year of life), genetic factors (especially HLADRB1 * 15) and EBV serostatus [3]. In recent years modifiable environmental factors have moved into the forefront of research, especially those supporting the development of MS. These include smoking, table salt consumption - although evidence for table salt is so far based on experimental data [4], higher body-mass index in childhood, low level of vitamin $D$ as well as alcohol consumption [5]. These factors are of particular interest since they are useful in the sense of primary or secondary prophylaxis, i.e. they can be modified easily or at least without major side effects by changing lifestyle. It should be noted, however that a) there are no modern intervention studies on the effects of a changed lifestyle on MS risk or on the course of the disease, and b) changing a susceptibility factor for existing MS does not automatically have a positive effect. However, it is noticeable that almost all modifiable lifestyle risk factors of MS can come into contact with the adaptive immune system at boundary areas such as the lung or intestine. In recent years, the role of both organs has therefore been investigated more closely especially with respect to autoimmunity in the CNS [6, 7]. 


\section{Gut and Microbiome}

The gut plays a special role regarding orally-ingested substances such as vitamin $D$, salt and dietary fats. The human intestine, with an overall length greater than 5.5 meters and a surface area of approx. $35 \mathrm{~m}^{2}$ is in direct contact with the "environment". Due to the variety of microorganisms in the intestinal lumen (intestinal microbiome) this organ has an additional characteristic. With increasing optimization of the sequencing possibilities of genetic information, so-called next generation sequencing, it is now possible to dissect the microbiome through its genetics. This offers the possibility of identifying bacteria using means that were not available through classical microbiological methods. Sequencing of 16sRNA also allows the identification of individual bacterial strains. It is known that up to 100 times more bacterial genes are found in the human body compared to human genetic information [8]. Much of this microbiome is attributable to the genetic material of the intestinal flora. The digestive tract contains a wide variety of germs of approx. $10^{14}$ bacteria. The distribution is $\mathrm{pH}$-dependent and with a population of $10^{1}$ is lower in the stomach (lactobacilli, streptococci) moderately distributed at $10^{3}, 10^{4}$ and $10^{7}$ in the duodenum, jejeunum and ileum respectively (as well as lactobacilli, E. coli, enterococcus faecalis), and quantitatively more widely pronounced in the colon with a population of $10^{12}$ (primarily lactic acid bacteria, bacteroids, and bifidobacterium) [9]. The microbiome is subject to an age-dependent change with already distinct differences between newborns fed by the breast or bottle, or those delivered by Cesarean section compared to vaginal delivery [10]. Further differences are found between normal and overweight adults as well in those who are older [11].

\section{The Role of the Gut During Illness and Interaction with the Immune System}

A distinction is made among intestinal germs: those that are useful for humans (symbionts with beneficial effects on the immune system), germs without deleterious effect (commensal) and those that have potentially pathological significance (pathobionts which can contribute to inflammatory processes). Under physiological conditions, there is a balance between symbionts and pathobionts, or between regulatory (e.g., regulatory T cells, Treg) and pro-inflammatory influences (Th1 and Th17 responses) on the immune system. Microbial imbalance in the intestine with a preponderance of pathobionts and pro-inflammatory influences is called dysbiosis and may be triggered by exogenous pathogens, for example. Numerous factors can ultimately contribute to dysbiosis, the genetic environment of the host (e. g., mutations in the genes for NOD2, IL23R, IGRM), lifestyle (stress, diet-related factors, etc.) bacterial colonization in early life (hospital birth, Cesarean section, breast feeding, etc.) [12] as well as iatrogenic influences (hygiene, inoculations, antibiotics) [13].

In the gut, due to its immediate proximity, intestinal bacteria closely interact with the immune system located in the intestinal wall. In fact, the intestine is an important immune organ in the body and includes central cell types and mediators of the congenital as well as the acquired immune system. In addition to macrophages and dendritic cells as antigen presenting cells (APC), lymphocytes are found in the lamina propria (so-called GALT, gut associated lym- phatic tissue, which are especially evident as Peyer plaques in the small intestine) and in the mesenteric lymph nodes. Germs of the intestinal lumen can communicate with these lymphocyte populations via the presentation of cell wall components via APC, directly via soluble factors or indirectly via the stimulation and modulation of intestinal epithelial cells. Promotion of regulatory T cell responses via the regulatory cytokine interleukin (IL)-10 can thereby balance pro-inflammatory $T$ helper responses with production of IL-17, IL-23 and tumor necrosis factor alpha [14]. A disbalance between Treg and Th17 cells in the immune system with prevailing pro-inflammatory Th17/Th1 responses is found in some autoimmune disorders just as in MS. In 2010 it was demonstrated for the first time that so-called segmented filamentous bacteria (SFB) of the intestine are able to drive autoimmune diseases such as in the case of rheumatoid arthritis via the activation of Th17 cells [15]. It therefore seems quite conceivable that changes in the intestine with imbalance of the immune system and increased activation of Th1/Th17 cells also play an important role in MS. This hypothesis was the starting point for characterizing the role of the intestine and also the microbiome with respect to MS.

\section{Significance of Gut and Microbiome for MS and in the MS Model}

First studies on the role of the gut in the experimental MS model, the so-called autoimmune experimental encephalomyelitis (EAE), used antibiotics to alter the intestinal microbiome [16]. For example, administration of neomycin or a kanamycin-based treatment regimen resulted in a weakened or delayed course of EAE in most studies. Since some of the antibiotics used may also have direct effects on immune cells or the CNS itself (e. g., minocycline), many of these studies have only limited validity regarding the specific role of the gut for autoimmunity in the CNS. Studies by the Max Planck Institute for Neurobiology in Munich provided an important breakthrough in 2011 based on a genetically modified mouse model. Using completely germ-free mice (gnotobiotic) in transfer experiments, it was clearly demonstrated that intact gut flora, at least in an experimental model, is an absolute prerequisite for the development of an autoimmune demyelination in the CNS [7]. These sophisticated experiments resulted in the concept that in the case of MS, misselected autoreactive myelin-resistant T cells in the thymus can be activated in the gut, and after recirculation in the immune system acquire migratory characteristics allowing them to enter the CNS, resulting in inflammatory demyelination [17].

While these important studies demonstrate the prominent role of the gut and intestinal microbiome in chronic inflammatory CNS disorders, the question of a specific bowel germ which could be responsible for the MS remained open. In the past two years basic studies have been published on changes to the microbiome in MS patients. A first Japanese study shows a reduction of Clostridia species in the intestinal microbiome of MS patients (Clostridium clusters XIVa and IV and Bacteroides) [18]. A recent Harvard study, on the other hand, shows an increase in Methanobrevibacter and Akkermansia as well as a decrease of Butyricimonas in the intestinal microbiome of MS patients [19]. A parallel study from the Mayo Clinic shows an increased abundance of Mycoplana, Haemophilus, 
Blautia and Dorea genera in MS patients [20]. Regarding pediatric MS, a Canadian working group found an increased abundance of Desulfovibrionaceae as well as a depletion of Lachnospiraceae and Ruminococcaceae among patients with active MS [21]. It is striking that all studies found different microbiome changes and no uniform pattern could be detected among the different cohorts. Whether this discrepancy is based on purely methodological reasons, or a defined germ associated with MS cannot be determined at this time. It should be critically noted that these studies of the microbiome in MS patients are purely descriptive and apart from demonstration of altered intestinal permeability in these patients [22], there have been few published functional analyses of the gut in MS. Discrepant results and lack of functional reappraisal are limited not only to the microbiome and MS field, but also to other autoimmune diseases for which a pathogenic association with the intestinal microbiome is suspected, e. g., diabetes mellitus [23].

Solely in the case of neuromyelitis optica (NMO) could a functional relationship be found between changes in the microbiome and immune system. An early study showed over-representation of C. perfringens in the intestinal microbiome. In fact, there is a structural homology of a clostridial ATP cassette-binding protein with astroglially expressed aquaporin-4 (AQP-4). AQP-4 plays a central role for $T$ and $B$ cell responses in the immunopathogenesis of $\mathrm{NMO}$, so mechanism of molecular mimicry appears conceivable [24].

\section{Intestine and Saturated Fatty Acids in MS: the Role of Priopionate}

The results of the purely quantitative analysis of individual gut germs in MS have so far provided no clear insights into the mechanism. It is therefore all the more interesting to consider the role of the intestinal microbiome in the pathomechanism of MS not only in terms of the pure number of individual germs, but rather their metabolic products. This field of "metabolomics" is currently attracting a lot of attention in science, especially from the perspective that dietary factors can have a significant impact on microbial metabolism. Thus a fiber-rich diet leads to the production of shortchain saturated fatty acids in the intestine which have beneficial effects on the intestinal epithelium and also directly on immune cells [25]. In contrast, long-chain polyunsaturated fatty acids such as omega 3 fatty acids have an anti-inflammatory effect and in metabolic models are shown to have anti-diabetic characteristics. Although a randomized Norwegian multi-center study in 2012 demonstrated no overall positive effects on relapsing-remitting MS for omega 3 fatty acids [26], we recently studied the effects of short-chain saturated fatty acids in an MS model [27]. It was known from metabolic models that the formation of short-chain saturated fatty acids acetate, butyrate and propionate from a fiber-rich diet have a positive effect on glucose metabolism via the portal vein and the CNS [28]. At the same time, epidemiological MS studies and microbiome analyses of a mouse indicate that the investigation of long-chain saturated fatty acids appear to be useful. In a humanized gnotobiotic mouse model, the change from a healthy, low-fat diet to a fat-rich diet ("Western diet") had a significant impact on the composition of the microbiome within one day as well as on the microbial metabolic pathways [29]. In addition, various

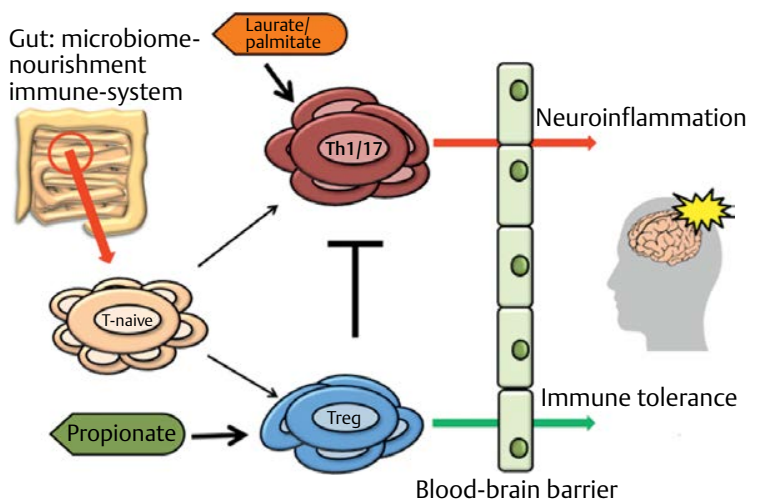

- Fig. 1 Dietary fatty acids ( $L A=$ laurate; $P A=$ propionate) act via the gut microbiome on gut resident $T$ cells that circulate and in the case of MS eventually access the central nervous system. Long chain fatty acids (LCFA) such as LA promote the differentiation of pro-inflammatory $T$ cells (such as Th17); in contrast short chain fatty acids (SCFA) such as PA lead to increased regulatory cell differentiation (Treg), which may inhibit inflammatory cells and impact the disease in a positive manner.

studies on independent cohorts have shown that overweight in adolescents, expressed by increased body mass index, increases the risk of MS in boys and girls [30,31]. An older study published in The Lancet demonstrated initial evidence for the positive effects of a diet with a lower proportion of saturated long-chain fatty acids (less than $20 \mathrm{~g} /$ day) in a smaller study group of MS patients [32].

In a study of our own we investigated the immune response to the effect of medium-chain, saturated fatty acids, such as laurate and palmitate that are contained in a variety of foods such as chocolate-based sandwich spread [27]. The addition of laurate to T cells in culture increased the frequency of pro-inflammatory Th $1 /$ Th 17 cells and reduced the frequency of regulatory $T$ cells. Similar observations were found in vivo after feeding a laurate-rich diet in the MS model with an increased frequency of pro-inflammatory Th1/ Th17 cells in the CNS as well as in the gut. While the analysis of the intestinal microbiome demonstrated slight changes in the model, metabolic studies of a laurate-rich diet showed a reduction in the short-chain saturated fatty acids acetate, butyrate and propionate. Additional functional studies with propionate showed that addition to $T$ cells in culture increased the frequency of regulatory $T$ cells while simultaneously reducing the frequency of pro-inflammatory Th17 cells. Similar observations were found in vivo after administration of propionate in the MS model with a moderated course, a reduction of demyelination and axon damage and the detection of functionally competent regulatory $T$ cells in the gut ( Fig. 1). In gnotobiotic mice, feeding short-chain saturated fatty acids also demonstrated positive effects on morphological changes and function of microglia [33], thus indicating propionate-dependent effects on other cell types beyond T cells.

Propionate is found mainly in non-digestible form in fiber-rich food, therefore bacterial action in the intestine is necessary for its release. Until the 1990s propionate was used as a preservative in bread products. The substance is approved both in Europe (EFSA) and the USA (FDA) as a food additive and can be purchased in apoth- 
ecaries. After initial positive immunological studies in both subjects and MS patients, the substance could represent a useful addition as an add-on therapy to the approved immunotherapeutic agents in MS and further studies in autoimmune diseases are eagerly awaited.

\section{SUMMARY AND OUTLOOK}

In summary, recent studies have shown an important role for the gut and especially the intestinal microbiome in the development and possibly therapy of MS. However, the research results so far also reveal a very differentiated picture of the complex interplay of the gut, microbiome, diet and immune system. Identification of a single "MS germ" or the call for a "gut renovation" as therapy for MS appear to be unrealistic in the short term. Caution should be used prior to the uncritical use of imbalanced diets, long-term antibiotic treatment to influence the microbiome or fecal transplant (effective in the case of pseudomembranous colitis) for causal therapy outside of well-considered studies. However, the advancement of functional analyses on the role of diet, intestines and immune systems in MS can contribute to the characterization of new, pathogenically relevant signaling pathways, and - as in the case of propionate - identification of new prospects for innovative therapies are awaited with interest.

\section{Conflict of Interest}

The authors declare no conflicts of interest.

\section{References}

[1] Galton F. On men of science, their nature and their nurture. Proceedings of the Royal Institution of Great Britain; 1874

[2] Haghikia A, Hohlfeld R, Gold R et al. Therapies for multiple sclerosis: translational achievements and outstanding needs. Trends Mol Med 2013; 19: 309-319

[3] Ascherio A, Munger KL, Lunemann JD. The initiation and prevention of multiple sclerosis. Nat Rev Neurol 2012; 8: 602-612

[4] Ascherio A, Munger KL. People with MS should consume a low-salt diet - NO. Mult Scler 2016; 22: 1779-1781

[5] Jorg S, Grohme DA, Erzler M et al. Environmental factors in autoimmune diseases and their role in multiple sclerosis. Cell Mol Life Sci 2016; 73: 4611-4622

[6] Odoardi F, Sie C, Streyl $\mathrm{K}$ et al. T cells become licensed in the lung to enter the central nervous system. Nature 2012; 488: 675-679

[7] Berer K, Mues M, Koutrolos M et al. Commensal microbiota and myelin autoantigen cooperate to trigger autoimmune demyelination. Nature 2011; 479: 538-541

[8] Sonnenburg JL, Backhed F. Diet-microbiota interactions as moderators of human metabolism. Nature 2016; 535: 56-64

[9] Tsabouri S, Priftis KN, Chaliasos N et al. Modulation of gut microbiota downregulates the development of food allergy in infancy. Allergol Immunopathol 2014; 42: 69-77

[10] Dominguez-Bello MG, Costello EK, Contreras M et al. Delivery mode shapes the acquisition and structure of the initial microbiota across multiple body habitats in newborns. Proc Natl Acad Sci U S A 2010; 107: 11971-11975
[11] Turnbaugh PJ, Hamady M, Yatsunenko T et al. A core gut microbiome in obese and lean twins. Nature 2009; 457: 480-484

[12] Gensollen T, lyer SS, Kasper DL et al. How colonization by microbiota in early life shapes the immune system. Science 2016; 352: 539-544

[13] Round JL, Mazmanian SK. The gut microbiota shapes intestinal immune responses during health and disease. Nat Rev Immunol 2009; 9: 313-323

[14] Ochoa-Reparaz J, Mielcarz DW, Begum-Haque S et al. Gut, bugs, and brain: Role of commensal bacteria in the control of central nervous system disease. Ann Neurol 2011; 69: 240-247

[15] Wu HJ, Ivanov II, Darce J et al. Gut-residing segmented filamentous bacteria drive autoimmune arthritis via T helper 17 cells. Immunity 2010; 32: 815-827

[16] Ochoa-Reparaz J, Mielcarz DW, Ditrio LE et al. Role of gut commensa microflora in the development of experimental autoimmune encephalomyelitis. J Immunol 2009; 183: 6041-6050

[17] Wekerle H. Nature plus nurture: the triggering of multiple sclerosis. Swiss Med Wkly 2015; 145: w14189

[18] Miyake S, Kim S, Suda W et al. Dysbiosis in the gut microbiota of patients with multiple sclerosis, with a striking depletion of species velonging to clostridia XIVa and IV cClusters. PLoS One 2015; 10: e0137429

[19] Jangi S, Gandhi R, Cox LM et al. Alterations of the human gut microbiome in multiple sclerosis. Nat Commun 2016; 7:12015 doi: $10.1038 /$ ncomms 12015

[20] Chen J, Chia N, Kalari KR et al. Multiple sclerosis patients have a distinct gut microbiota compared to healthy controls. Sc Rep 2016: 28484 doi:10.1038/srep28484

[21] Tremlett H, Fadrosh DW, Faruqi AA et al. Gut microbiota composition and relapse risk in pediatric MS: A pilot study. J Neurol Sci 2016; 363: 153-157

[22] Buscarinu MC, Cerasoli B, Annibali V et al. Altered intestinal permeability in patients with relapsing-remitting multiple sclerosis: A pilot study. Mult Scler 2016; 23: 442-446

[23] Knip M, Siljander $\mathrm{H}$. The role of the intestinal microbiota in type 1 diabetes mellitus. Nat Rev Endocrinol 2016; 12: 154-167

[24] Cree BA, Spencer CM, Varrin-Doyer M et al. Gut microbiome analysis in neuromyelitis optica reveals overabundance of Clostridium perfringens. Ann Neurol 2016; 80: 443-447

[25] Maslowski KM, Mackay CR. Diet, gut microbiota and immune responses. Nat Immunol 2011; 12: 5-9

[26] Torkildsen O, Wergeland S, Bakke $S$ et al. omega-3 fatty acid treatment in multiple sclerosis (OFAMS Study): A randomized, double-blind, placebo-controlled trial. Arch Neurol 2012; 69: 1044-1051

[27] Haghikia A, Jorg S, Duscha A et al. Dietary fatty acids directly impact central nervous system autoimmunity via the small intestine. Immunity 2015; 43: 817-829

[28] Brussow H, Parkinson S]. You are what you eat. Nat Biotechnol 2014; 32: 243-245

[29] Turnbaugh P], Ridaura VK, Faith J] et al. The effect of diet on the human gut microbiome: A metagenomic analysis in humanized gnotobiotic mice. Sci Transl Med 2009; 1: 6ra14

[30] Munger KL, Chitnis T, Ascherio A. Body size and risk of MS in two cohorts of US women. Neurology 2009; 73: 1543-1550

[31] Hedstrom AK, Lima Bomfim I, Barcellos L et al. Interaction between adolescent obesity and HLA risk genes in the etiology of multiple sclerosis. Neurology 2014; 82: 865-872

[32] Swank RL, Dugan BB. Effect of low saturated fat diet in early and late cases of multiple sclerosis. Lancet 1990; 336: 37-39

[33] Erny D, Hrabe de Angelis AL, Jaitin D et al Host microbiota constantly control maturation and function of microglia in the CNS. Nat Neurosci 2015; 18: 965-977 